\title{
Design of Few-Mode Fibers with Arbitrary and Flattened Differential Mode Delay
}

\author{
Filipe Ferreira, Student Member, IEEE, Daniel Fonseca, and Henrique Silva, Member, IEEE
}

\begin{abstract}
This letter proposes the use of a refractive index profile with a graded core and a cladding trench for the design of few-mode fibers (FMFs), aiming an arbitrary differential mode delay (DMD) flattened over the $\mathrm{C}+\mathrm{L}$ band. By optimizing the core grading exponent and the dimensioning of the trench, a deviation lower than $0.01 \mathrm{ps} / \mathrm{km}$ from a target DMD is observed over the investigated wavelength range. Additionally, it has been found that the dimensioning of the trench is almost independent of the target DMD, thereby enabling the use of a simple design rule which guarantees a maximum DMD deviation of $1.8 \mathrm{ps} / \mathrm{km}$ for a DMD target between $-200 \mathrm{ps} / \mathrm{km}$ to $200 \mathrm{ps} / \mathrm{km}$.
\end{abstract}

Index Terms-Few-Mode Fibers, Differential Mode Delay, Refractive Index Profile.

\section{INTRODUCTION}

$\mathrm{F}$ EW MODE FIBERS (FMFs) have been investigated in the last years due to their larger information transport capacity, when compared with single mode fibers (SMFs) [1]. An $N$-fold capacity increase, with $N$ equal to the number of independent modes of the FMF, is obtained using mode division multiplexing. However, such increase is only attained at the cost of higher complexity of the equalizer, as it needs to compensate for the combined effect of differential mode delay (DMD) and modal crosstalk, which originates a channel impulse response spread over a long time interval [2]. In order to relax the complexity of the equalizer, two different approaches to maximize the multi-mode transmission reach were proposed: the use of FMFs with inherently low DMD [4], and the use of DMD compensated FMFs [5] (FMFs with positive DMD and FMFs with negative DMD, concatenated). According to the literature, the FMF with lowest DMD reported up to date has a DMD between the two supported linearly polarized (LP) modes, LP01 and LP11 (composed by two degenerate modes, LP11a and LP11b), of $4.4 \mathrm{ps} / \mathrm{km}$ for a

Manuscript received June 1, 2012. This work has been partially supported by: Nokia Siemens Networks S.A. Portugal, the Fundação para a Ciência e Tecnologia (FCT) under the grant SFRH/BDE/51094/2010, and the European Communities $7^{\text {th }}$ Framework Programme under grant agreement 258033 (MODE-GAP)

Filipe Ferreira and Daniel Fonseca are with Nokia Siemens Networks Portugal, S. A., Amadora and with Instituto de Telecomunicações, Portugal (email: filipe.ferreira@nsn.com and daniel.fonseca@nsn.com).

Henrique Silva is with Instituto de Telecomunicações, University of Coimbra, Portugal (email: hjas@co.it.pt).

Copyright (c) 2012 IEEE. Personal use of this material is permitted. single wavelength $1550 \mathrm{~nm}$ [4]. However, for the transmission of wavelength-multiplexed signals a low DMD is required over a broad wavelength range, and thus the DMD average and slope have to be as low as possible. Regarding DMD compensated FMFs, [5] reported a residual LP01-LP11 DMD lower than $6 \mathrm{ps} / \mathrm{km}$ in the $\mathrm{C}$ band after $100 \mathrm{~km}$ of transmission, at the cost of supporting modes with order higher than the LP11, which severely limits the transmission distance. In this case, the use of FMFs with non-zero and symmetric DMD slope is as effective in the reduction of the residual DMD as using FMFs with zero DMD slope. In this letter we consider only the optimization of FMFs for zero slope, since this is as challenging as obtaining it for any given finite slope.

In this work, our objective was to design FMFs supporting only two LP modes, with the lowest possible DMD slope over the $\mathrm{C}+\mathrm{L}$ band. For that we considered a refractive index profile with a graded core and a cladding trench, as already used in the past for the design of chromatic dispersionflattened single-mode fibers [6]. The impact on the DMD (average and slope) of the core grading exponent and of the dimensioning of the trench is described in Section II, as well as the parameters search domains and the optimizing function. Section III presents the optimization results for three target DMD values: $0 \mathrm{ps} / \mathrm{km}, 100 \mathrm{ps} / \mathrm{km}$ and $-100 \mathrm{ps} / \mathrm{km}$. The results show that it is possible to reduce the DMD difference to the target DMD to less than $0.01 \mathrm{ps} / \mathrm{km}$, and that the dimensioning of the trench is almost independent of the target DMD. Therefore, a simple design rule has been derived, which guarantees a maximum DMD deviation over the $\mathrm{C}+\mathrm{L}$ band lower than $1.8 \mathrm{ps} / \mathrm{km}$ for a DMD target between $-200 \mathrm{ps} / \mathrm{km}$ to $200 \mathrm{ps} / \mathrm{km}$. Additionally, tolerance of the designed FMFs to manufacturing margins was evaluated and found to be insufficient. The main conclusions are gathered in section IV.

\section{FIBER PROFILE DESCRIPTION, ANALYSIS AND OPTIMIZATION}

The refractive index profile under consideration, composed by a graded core and a cladding trench, is described by:

$$
n(\rho)=\left\{\begin{array}{ccc}
n(0)\left[1-\Delta n_{c o}\left(\rho / w_{1}\right)^{\alpha}\right], & |\rho|<w_{1} \\
n_{c l}, & w_{1} \leq|\rho|<w_{1}+w_{2} \\
n_{c l} /\left(1-\Delta n_{t r}\right), & w_{1}+w_{2} \leq|\rho|<w_{1}+w_{2}+w_{3} \\
n_{c l}, & |\rho| \geq w_{1}+w_{2}+w_{3}
\end{array}\right.
$$

where $n(\rho)$ is the refractive index as function of the radial distance $(\rho)$ and $n_{c l}$ is the cladding refractive index. $w_{1}$ is the 
core radius, $w_{2}$ is the radial distance between the end of the core and the beginning of the trench, and $w_{3}$ is the trench width. $\Delta n_{c o}$ is the relative refractive index difference $(\Delta n)$ at $\rho=0$ and $\Delta n_{t r}$ is $\Delta n$ at the trench, with $\Delta n(\rho)$ given by: $\Delta n(\rho)=\left[n(\rho)-n_{c l}\right] / n(\rho)$. During the optimization for each different combination of these parameters, the guided modes supported by the fiber and their characteristics, as well as the effective group index $\left(\bar{n}_{g}\right)$ were calculated by solving the Maxwell equations numerically [7] using the Runge-Kutta method. The dispersion properties of the doped silica have been modeled by using the Sellmeier coefficients provided in [8].

There are two parameters in (1) that can be defined without optimization: $w_{1}$ and $\Delta n_{c o} . w_{1}$ should be as large as possible in order to reduce the nonlinear coefficients for all modes. Therefore, the highest core radius reported to date for a FMF, $w_{1}=9 \mu \mathrm{m}$, was considered [9]. In order to determine $\Delta n_{c o}$, we have calculated the highest value of the normalized frequency $V$ that guarantees the support of only two LP modes for $\alpha=2$ and $\Delta n_{t r}=0$, which is $V=5$.1. Since $V=2 \pi w_{1} / \lambda \cdot\left[n(0)^{2}-n_{c l^{2}}\right]^{1 / 2}$, $\Delta n_{c o}$ was found to be $4.5 \cdot 10^{-3}$, for the lowest $\lambda$ of the $\mathrm{C}+\mathrm{L}$ band.

In order to understand the impact of the core grading exponent and of the trench dimensioning on the DMD, one has to consider that the DMD is proportional to the difference between the values of $\bar{n}_{g}$ for the supported modes LP01 $\left(\bar{n}_{g}{ }^{\mathrm{LP} 01}\right)$ and LP11 $\left(\bar{n}_{g}^{\mathrm{LP} 11}\right): D M D(\lambda)=L / c \cdot\left[\bar{n}_{g}^{\mathrm{LP} 11}(\lambda)-\bar{n}_{g}^{\mathrm{LP} 01}(\lambda)\right]$, where $L$ is the fiber length and $c$ is the light velocity in vacuum. The $\bar{n}_{g}$ of a mode depends on the fraction of the mode power that propagates in each region of the fiber cross section. Thus, a tightly confined mode has a $\bar{n}_{g}$ that is dominated by the group index of the core (as the LP01). On the other hand, a weakly confined mode will propagate with a smaller $\bar{n}_{g}$, closer to the group index of the cladding (as the LP11, since the confinement decreases with the mode order).

Based on the explanation presented in the last paragraph, we started by analyzing the impact of $\alpha$ on $\bar{n}_{g}$, in order to understand its impact on the DMD. Fig. 1 a) presents $\bar{n}_{g}$ for the LP01 and LP11 modes as a function of the wavelength and $\alpha$, in the absence of trench. In Fig. 1 a) it can be seen that the difference between the $\bar{n}_{g}^{\mathrm{LP} 11}$ and $\bar{n}_{g}^{\mathrm{LP} 01}$ curves, which is proportional to the DMD, decreases with increasing $\alpha$, and that the decrease seems to vary only slightly in the $\mathrm{C}+\mathrm{L}$ band, since the $\bar{n}_{g}{ }^{\mathrm{LP} 01}$ and $\bar{n}_{g}^{\mathrm{LP} 11}$ curves for different values of $\alpha$ are almost parallel. To understand this effect, the inset in Fig. 1 a) presents the normalized amplitude of the field for the LP01 and LP11 modes as a function of $\rho / w_{1}$ and $\alpha$. It shows that with increasing $\alpha$ the power confinement of the LP01 mode in the core decreases slightly, compared to the increase of the power confinement of the LP11 mode. This, combined with the increase of the group index in the core itself, is sufficient for the increase of $\bar{n}_{g}$ for both modes. This increase is higher for $\bar{n}_{g}{ }^{\mathrm{LP} 11}$, due to the slight decrease of the power confinement for the LP01 mode, and thus the DMD decreases.

In order to assess the impact of the trench on $\bar{n}_{g}$, and thus on the DMD, Fig. $1 \mathrm{~b}$ ) presents $\bar{n}_{g}$ as a function of the wavelength and $\Delta n_{t r}$. As can be seen in Fig. $1 \mathrm{~b}$ ), the trench affects mainly
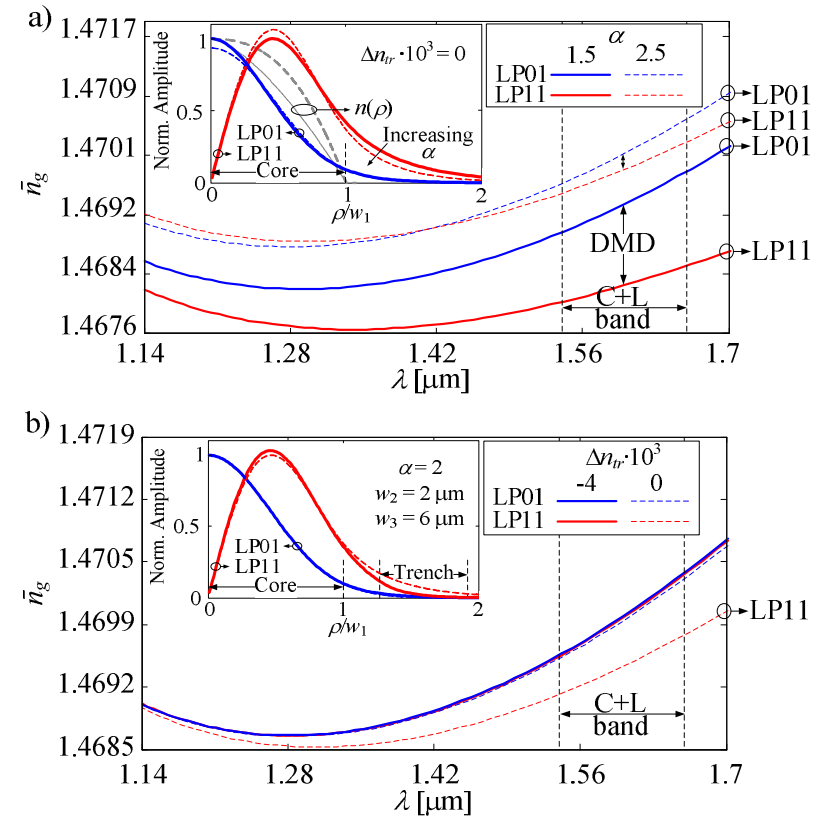

Fig. 1. $\bar{n}_{g}$ of the LP01 and of the LP11 as a function of $\lambda$ : a) varying $\alpha, \mathrm{b}$ ) varying $\Delta n_{t r}$. Inset a) and b): Normalized amplitude of the field for the LP01 and LP1 1 modes as a function of $\rho / w_{1}$, considering $\lambda=1550 \mathrm{~nm}$.

the LP11 mode and allows controlling the DMD slope. This can be understood by looking into the field distribution. The inset in Fig. $1 \mathrm{~b}$ ) presents the normalized amplitude of the field for the LP01 and LP11 modes as a function of $\rho / w_{1}$ and $\Delta n_{t r}$. It shows that the power confinement of the LP11 mode in the core increases with decreasing $\Delta n_{t r}$ (creating a deeper trench), and that the field distribution of the LP01 mode is only slightly affected, so the DMD decreases. Furthermore, the increase with wavelength of the mode field radius for the LP11 mode is counteracted by the power confinement effect of the trench. Thus, the trench has the effect of flattening the DMD over a certain wavelength band, with an adequate trench dimensioning.

In order to search for the optimum $\alpha$ and dimensioning of the trench $\left(\Delta n_{t r}, w_{2}, w_{3}\right)$, an optimization function was introduced with the objective of minimizing the magnitude of the DMD error relative to the DMD target ( $\psi$ ) over a defined $\lambda$ range:

$F O\left(\alpha, \Delta n_{t r}, w_{2}, w_{3}\right)=\frac{\int_{\lambda^{-}}^{\lambda^{+}}\left|D M D\left(\lambda, \alpha, \Delta n_{t r}, w_{2}, w_{3}\right)-\psi\right| d \lambda}{\lambda^{+}-\lambda^{-}}$

The minimization of the integral of the modulus of the DMD deviation from $\psi$ forces its reduction over the whole wavelength range. This objective function is subject to the following constraints:

$$
\begin{gathered}
\left(\alpha^{-}=1.5\right) \leq \alpha \leq\left(\alpha^{+}=2.5\right) \\
\left(\Delta n_{t r}{ }^{-}=-0.0045\right) \leq \Delta n_{t r} \leq\left(\Delta n_{t r}{ }^{+}=0\right) \\
\left(w_{2}{ }^{-}=0\right) \leq w_{2} \leq\left(w_{2}{ }^{+}=w_{1} / 2\right) \\
\left(w_{3}{ }^{-}=0\right) \leq w_{3} \leq\left(w_{3}{ }^{+}=w_{1}\right) \\
\left(\lambda^{-}=1530 \cdot 10^{-9}\right) \leq \lambda \leq\left(\lambda^{+}=1625 \cdot 10^{-9}\right)
\end{gathered}
$$

The search domain for $\alpha$, defined by (3), was selected since it is well known that $\alpha \approx 2$ minimizes the DMD for multi-mode 
fibers [10]. (4) constrains $\Delta n_{t r}$ to values higher than -0.0045 , since a deeper trench would be difficult and expensive to fabricate [6]. In (5), $w_{2}$ is limited to $w_{1} / 2$ as a trench farther away from the core has reduced or none effect on the core modes. (6) constrains $w_{3}$ to values smaller than $w_{1}$, since a wider trench would make the fabrication more expensive. The wavelength domain (7) is bounded to the $\mathrm{C}+\mathrm{L}$ window.

\section{RESULTS AND DISCUSSION}

\section{A. Fiber Profile Optimization}

In this sub-section we consider three different $\psi$ values: $0 \mathrm{ps} / \mathrm{km}, 100 \mathrm{ps} / \mathrm{km}$ and $-100 \mathrm{ps} / \mathrm{km}$. In order to find the $\left(w_{2}, w_{3}\right)$ pairs that allow the reduction of the DMD slope to negligible levels, Fig. 2 shows the contour map of the minimum FO $[\mathrm{ps} / \mathrm{km}]$ as a function of $w_{2}$ and $w_{3}$ for $\psi=0 \mathrm{ps} / \mathrm{km}$. For each pair $\left(w_{2}, w_{3}\right)$, the optimum pair $\left(\alpha, \Delta n_{t r}\right)$ that minimizes FO is used. A subset of the domain of $w_{2}$ and $w_{3}$, defined by (5) and (6), is shown in order to improve the visualization of the FO variation around the optimum values. The shaded area covers values where FO is obtained for optimum $\Delta n_{t r}<-4.5 \cdot 10^{-3}$ and, therefore, out of the domain defined in section II.

Fig. 2 shows FO below $0.1 \mathrm{ps} / \mathrm{km}$ for a clearly delimited area, with $w_{2}$ ranging from $\sim 1.7 \mu \mathrm{m}$ to $\sim 2 \mu \mathrm{m}$ and $w_{3}$ from $\sim 4.5 \mu \mathrm{m}$ to $9 \mu \mathrm{m}$. Additionally to $\mathrm{FO}$, the maximum DMD deviation (MDD) relatively to the targeted DMD over the $\mathrm{C}+\mathrm{L}$ band, $\operatorname{MDD}\left(\alpha, \Delta n_{t r}, w_{2}, w_{3}\right)=\max \lambda\left|D M D\left(\lambda, \alpha, \Delta n_{t r}, w_{2}, w_{3}\right)-\psi\right|$, was calculated, since FO is an indirect measure of the DMD. We have chosen to calculate the MDD instead of the DMD slope, as in [5], because the accuracy of the MDD does not depend on the linearity of the DMD. In the case of the FO minimum presented in Fig. 2 for $\psi=0 \mathrm{ps} / \mathrm{km}$, the MDD is $0.01 \mathrm{ps} / \mathrm{km}$, revealing an almost flattened DMD dependence with the $\lambda$.

Table I shows the FO minimum, the MDD and the corresponding optimum profile parameters for the three $\psi$ values. The minimum FO is below $0.004 \mathrm{ps} / \mathrm{km}$ and the MDD is below $0.01 \mathrm{ps} / \mathrm{km}$, for any of the $\psi$ values. The propagation characteristics of the designed fibers were also evaluated: the curvature radius $(R c)$ for a macrobending loss (MBL) of $0.5 \mathrm{~dB}$, calculated according to [11], the chromatic dispersion $(D)$, the chromatic dispersion slope $(S)$ and the nonlinear coefficient $(\gamma)$. The $R c$ values determined for the LP11 mode (the mode with higher MBL) are around $4.5 \mathrm{~mm}$, and thus

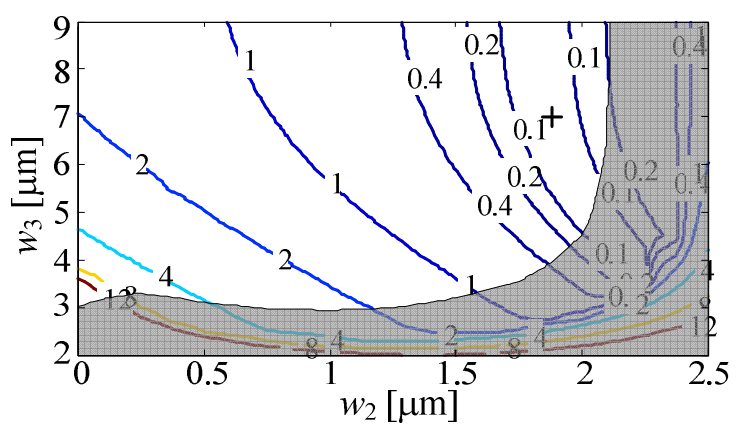

Fig. 2. Contour map of $F O[\mathrm{ps} / \mathrm{km}]$ as a function of $w_{2}$ and $w_{3}$, for $\psi=0 \mathrm{ps} / \mathrm{km}$. ' + ' marks the FO minimum, which is $0.004 \mathrm{ps} / \mathrm{km}$.
TABLE I

OPTIMIZED FIBER CHARACTERISTICS AND PARAMETERS.

\begin{tabular}{l|ccc}
\hline \hline & \multicolumn{3}{|c}{ DMD target [ps/km] } \\
& -100 & 0 & 100 \\
\hline$F O[\mathrm{ps} / \mathrm{km}]$ & 0.0005 & 0.0040 & 0.0019 \\
$M D D[\mathrm{ps} / \mathrm{km}]$ & 0.0017 & 0.0101 & 0.0050 \\
\hline$\alpha$ & 2.158 & 2.093 & 2.032 \\
$\Delta n_{t r} \cdot 10^{3}$ & -3.897 & -3.953 & -4.010 \\
$w_{2}[\mu \mathrm{m}]$ & 1.875 & 1.875 & 1.875 \\
$w_{3}[\mu \mathrm{m}]$ & 8.750 & 7.00 & 6.250 \\
\hline \hline
\end{tabular}

below the value of $7.5 \mathrm{~mm}$ specified by [12] for all cases. The $D$ values are around $22 \mathrm{ps} / \mathrm{km} / \mathrm{nm}$, only slightly higher than the dispersion of $\sim 17 \mathrm{ps} /(\mathrm{nm} \cdot \mathrm{km})$ characteristic of standard singlemode fibers (SSMF). The $S$ values are around $0.06 \mathrm{ps} /\left(\mathrm{nm}^{2} \cdot \mathrm{km}\right)$, lower than $\sim 0.08 \mathrm{ps} /\left(\mathrm{nm}^{2} \cdot \mathrm{km}\right)$ typical of SSMF. The $\gamma$ value is around 1.04 for the LP01 and 0.75 for the LP11, for all cases, significantly lower than the SSMF typical value of 1.3 , as expected.

\section{B. Simple Design Rule}

In order to derive a simple design rule some of the profile parameters have to be fix. From Table I and Fig. 2 we can concluded that the optimum $\left(w_{2}, w_{3}\right)$ pair for the three $\psi$ values is inside the area delimited by FO $<0.1 \mathrm{ps} / \mathrm{km}$. Moreover, for the three $\psi$ values, additional results have shown that such area are overlap and that the $\Delta n_{t r}$ contour map as a function of $w_{2}$ and $w_{3}$ is approximately the same. Therefore, the optimum dimensioning of the trench can be detached from the optimization of $\alpha$, with a small DMD penalty. Given this, a simple design rule for the quasi-optimum $\alpha$ value as a function of $\psi$ can be derived. The motivation for the derivation of such design rule with a fixed set $\left(\Delta n_{t r}, w_{2}, w_{3}\right)$ is the simplification of the optimization procedure in the fabrication process, since only the concentrations of dopants in the core change between different DMD targets.

The first step in the derivation of the design rule was the dimensioning of the trench. The $\left(w_{2}, w_{3}\right)$ pair was determined minimizing FO simultaneously for the three targets, obtaining: $w_{2}=1.875 \mu \mathrm{m}$ and $w_{3}=7 \mu \mathrm{m}$. For this $\left(w_{2}, w_{3}\right)$ pair, the variation of the optimum $\Delta n_{t r}$ value for each of the targeted $\psi$ values is lower than $1.5 \%$, and the FO deviation from the optimum results shown in Table I is lower than $0.05 \mathrm{ps} / \mathrm{km}$. Therefore, the $\Delta n_{t r}$ chosen for the design rule was the optimum $\Delta n_{t r}$ for $\psi=0 \mathrm{ps} / \mathrm{km}: \Delta n_{t r} \cdot 10^{3}=-3.953$. Fig. 3 shows the optimum $\alpha$ and the respective MDD as a function of $\psi$. It can be seen from Fig. 3 that the variation of the optimum $\alpha$ with $\psi$ is approximately linear, and that the MDD is always lower than $1.8 \mathrm{ps} / \mathrm{km}$. By fitting a second order polynomial to the optimum $\alpha$ values, the equation shown in the inset of Fig. 3 was obtained. Using this equation, Fig. 3 shows the quasioptimum $\alpha$ line and the corresponding MDD line. From these lines it can be concluded that the fitting equation shown in the inset of Fig. 3 is sufficient for the design of a FMF with any DMD value from $-200 \mathrm{ps} / \mathrm{km}$ to $200 \mathrm{ps} / \mathrm{km}$, and a MDD lower than $1.8 \mathrm{ps} / \mathrm{km}$ over the $\mathrm{C}+\mathrm{L}$ band. Regarding the propagation 


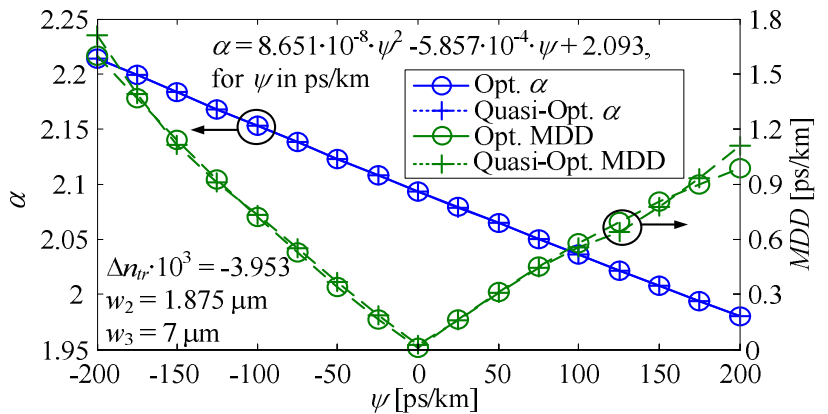

Fig. 3. Optimum $\alpha$ and MDD as a function of $\psi$.

characteristics $R c, D, S$ and $\gamma$, of the fibers designed following the given rule, their values are very similar to the values obtained with the optimum parameters given by a full optimization. Therefore, similar comments are applicable.

\section{Manufacturing Margins}

The optimized parameters presented in Table I and the parameters obtained from the simple design rule were obtained ignoring the finite precision margins of the manufacturing control processes. Those margins may lead to some deviation of the manufactured fiber parameters from the optimum values. The typical fabrication tolerance for $\Delta n_{c o}$ or $\Delta n_{t r}$ is \pm 0.0001 [13], for $w_{1}$ is $\pm 0.25 \mu \mathrm{m}$, and for $\alpha$ is \pm 0.01 [10]. These deviations result in a corresponding deviation of the DMD relative to the targeted value. In order to evaluate the tolerance to these deviations, the MDD value considered acceptable over the $\mathrm{C}+\mathrm{L}$ band is $12 \mathrm{ps} / \mathrm{km}$, since this is the DMD required for $2000 \mathrm{~km}$ of multi-mode transmission at $100 \mathrm{~Gb} / \mathrm{s}$ using CP-QPSK [3], for an overhead of up to $10 \%$.

Table II shows the parameters maximum tolerable deviation (MTD), such that MDD remains lower than the $12 \mathrm{ps} / \mathrm{km}$ requirement, for the optimum parameters of Table I and for the parameters given by the simple design rule presented in Section III B. The tolerance of each parameter was determined considering the remaining parameters fixed at their optimum values. The MTD values for $w_{1}, w_{2}$ and $w_{3}$ were obtained considering simultaneous and proportional deformation of $w_{1}$, $w_{2}$ and $w_{3}$ (independent deformations are also possible and can lead to less tolerance). Table II shows that the tolerance of any of the parameters is close or slightly below the manufacture margins, for all cases, except $\Delta n_{c o}$ for $\psi=-100 \mathrm{ps} / \mathrm{km}$. As no significant MDT difference is visible between the optimized values of section III A and the values obtained using the simple design rule, one concludes about the usefulness of the proposed simplification. Further results have shown that, for TABLE II PARAMETERS MAXIMUM TOLERABLE DEVIATION [\%].

\begin{tabular}{l|c|ccc|ccc}
\hline \hline & $\begin{array}{c}\text { Manufacturing } \\
\text { Margin }\end{array}$ & \multicolumn{2}{|c|}{ Full Optimization } & \multicolumn{2}{c}{ Simple Design Rule } \\
& DMD target [ps/km] & \multicolumn{2}{c}{ DMD target[ps/km] } \\
& -100 & 0 & 100 & -100 & 0 & 100 \\
\hline$\Delta n_{c o}$ & $\sim \pm 2.5$ & \pm 3.735 & \pm 2.676 & \pm 2.19 & \pm 3.885 & \pm 2.816 & \pm 2.229 \\
$w_{1}, w 2, w_{3}$ & $\sim \pm 2.5$ & \pm 2.358 & \pm 2.328 & \pm 2.289 & \pm 2.427 & \pm 2.316 & \pm 2.208 \\
$\alpha$ & $\sim \pm 0.5$ & \pm 0.577 & \pm 0.577 & \pm 0.577 & \pm 0.560 & \pm 0.577 & \pm 0.563 \\
$\Delta n_{t r}$ & $\sim \pm 2.5$ & \pm 2.002 & \pm 2.002 & \pm 2.002 & \pm 2.002 & \pm 2.002 & \pm 2.002 \\
\hline \hline
\end{tabular}

the case of a simultaneous drift of two or more parameters, the MTD is reduced by one half or more. These results demonstrate that a non-negligible number of pre-forms will produce FMFs with a MDD higher than $12 \mathrm{ps} / \mathrm{km}$, resulting in an increase of the fabrication costs. Therefore, an improvement of the fabrication control process will be necessary. This problem can be partially circumvented by concatenating FMFs exhibiting positive and negative DMD deviations.

\section{CONCLUSIONS}

In this work a graded-index FMF with a cladding trench is proposed and optimized through simulation in order to obtain an arbitrary DMD flattened over the $\mathrm{C}+\mathrm{L}$ band. It has been shown that such design allows obtaining a MDD lower than $0.01 \mathrm{ps} / \mathrm{km}$ when $\alpha, \Delta n_{t r}, w_{2}, w_{3}$ are optimized. Furthermore, it has been concluded that the dimensioning of the trench is almost independent of the targeted DMD. A simple design rule for a quasi-optimum $\alpha$ has been derived guaranteeing a MDD over the $\mathrm{C}+\mathrm{L}$ band lower than $1.8 \mathrm{ps} / \mathrm{km}$, for a DMD target between $-200 \mathrm{ps} / \mathrm{km}$ and $200 \mathrm{ps} / \mathrm{km}$. However, the existing fabrication control process does not have enough precision to guarantee that every pre-form produces a FMF with a DMD lower than the $12 \mathrm{ps} / \mathrm{km}$ requirement. Nevertheless, the proposed profile is an excellent candidate to design FMFs with extremely low DMD.

\section{REFERENCES}

[1] R. Essiambre, and A. Mecozzi, "Capacity limits in single mode fiber and scaling for spatial multiplexing," in Proc. OFC'2012, USA, paper OW3D.1.

[2] A. Lobato, F. Ferreira, B. Inan, M. Kuschnerov, D. van den Borne, S. L. Jansen, B. Spinnler, and B. Lankl, "The Impact of Differential Mode Delay on Mode-Division Multiplexed Coherent Optical OFDM Transmission," in $O F C^{\prime} 2012$, USA, paper OTu2C.2.

[3] B. Inan, B. Spinnler, F. Ferreira, A. Lobato, S. Adhikari, et al, "Equalizer Complexity of Mode Division Multiplexed Coherent Receivers," in Proc. OFC'2012, USA, paper OW3D.4.

[4] L. Gruner-Nielsen, Y. Sun, J. Nicholson, D. Jakobsen, R. Lingle, and B. Palsdottir, "Few Mode Transmission Fiber with low DGD, low Mode Coupling and low Loss," in Proc. OFC'2012, USA, paper PDP5A.1.

[5] M. Li, B. Hoover, S. Li, S. Bickham, S. Ten, E. Ip, Y. Huan, E. Mateo, Y. Shao, and T. Wang, "Low delay and large effective area few-mode fibers for mode-division multiplexing," in Proc. OECC'2012, Korea, paper 5C3-2.

[6] B. Ainslie, and C. Day, "A review of single-mode fibers with modified dispersion characteristics," IEEE JLT, vol.4, no.8, pp.967- 979, 1986.

[7] J. G. Dil, and H. Blok, "Propagation of electromagnetic surface waves in a radially inhomogeneous optical waveguide," Opt. and Quant. Electron., vol. 5, no. 5, pp. 415-428, 1973.

[8] W.Hermann, and D.U.Wiechert, "Refractive index of doped and undoped PCVD bulk silica", Materials Research Bulletin, vol. 24, no.9, pp.1083-1097, 1989.

[9] K. Mukasa, K. Imamura, and R. Sugizaki, "Optimizing 2 mode fibers with Aeff of $170 \mu \mathrm{m}^{2}$ for LP01 mode and studying a possibility of realizing endlessly 2 mode operation using holey fibers," in Proc. $O F C$ '2012, USA, paper JW2A.16.

[10] P. Matthijsse, D. Molin, F. Gooijer, and Gerard Kuyt, "On the Design of Wide Bandwidth Window Multimode Fibers," in Proc. IWCS'2005, USA, pp.332-337.

[11] J. Sakai, and T. Kimura, "Bending loss of propagation modes in arbitrary-index profile optical fibers," OSA Appl. Opt., vol.17, no.10, pp.1499-1506, 1978.

[12] ITU-T recommendations G.657B, December 2006.

[13] A. Yablon, "Multi-Wavelength Optical Fiber Refractive Index Profiling by Spatially Resolved Fourier Transform Spectroscopy," IEEE JLT, vol. 28, no. 4, pp. 360-364, 2010. 